\title{
Concentration-Effect, Incidence and Mechanism of Nevirapine Hepatotoxicity
}

\author{
Adikwu Elias and Brambaifa Nelson \\ Department of Pharmacology, Faculty of Basic Medical Sciences, \\ College of Health Sciences, University of Port Harcourt, Choba, Rivers State, Nigeria
}

Received 2013-01-23, Revised 2013-03-24; Accepted 2013-04-19

\begin{abstract}
Nevirapine is a dipyridozepinone non nucleoside reverse transcriptase inhibitor approved for use in HIV-infected patients. Its efficacy has been well demonstrated in numerous clinical trials. It has activity against HIV-1 but does not have significant activity against HIV-2 or other retroviruses. Nevirapine is used as one of the components of highly active antiretroviral therapy in HIV patients and in the prevention of mother to child transmission of HIV. Reports have linked nevirapine containing highly active antiretroviral therapy with hepatotoxicity which is of great clinical concern. In this study, a comprehensive literature review on reports (previous and present) of nevirapine associated hepatotoxicity in experimental animal studies and humans (case reports, clinical trials and cohorts' studies) was performed. The relationships between length of nevirapine therapy, plasma concentration and nevirapine associated hepatotoxicity were evaluated. The possible mechanisms of nevirapine associated hepatotoxicity were also reviewed. Analysis of data from various clinical trials, cohort and other studies involving patients taking nevirapine containing antiretroviral therapy showed that nevirapine could be hepatotoxic. Nevirapine associated hepatotoxicity is of two distinguished types the early immune mediated hypersensitivity reaction which develops within 18 weeks and the late onset hepatotoxicity which occurs after 18 weeks of nevirapine therapy. Most nevirapine associated hepatotoxicity manifested as hepatitis, jaundice, cholestatic hepatitis, hepatic necrosis and fulminant hepatic failure with rare deaths. This hepatotoxicity is associated with elevation in levels of aspartate aminotransferase, alanine aminotransferase, alkaline phosphatase and other biomarkers of liver function. Liver enlargement and necrosis which occurred in centrilobular and mediolobular region marked with inflammations are features of nevirapine associated hepatotoxicity. Nevirapine hepatotoxicity might have correlation with length of therapy but with discrepancy in reports on the relationship between nevirapine hepatotoxicity and nevirapine plasma concentration. The mechanism of nevirapine associated hepatotoxicity is not well understood but could be attributed to direct toxic effect on the liver, mitochondrial dysfunction in hepatocytes, hypersensitivity reaction and stress induced by nevirapine. It is recommended that liver function should be evaluation for HIV patients eligible for nevirapine containing highly active antiretroviral therapy.
\end{abstract}

Keywords: Nevirapine, Toxicity, Mechanism, Plasma Concentration, Liver

\section{INTRODUCTION}

Human Immunodeficiency Virus (HIV) is a retrovirus known to be the primary cause of Acquired
Immune Deficiency Syndrome (AIDS). The HIV/AIDS epidemic has cause death in many countries. It is reported that about 39 million people globally are living with HIV. The high HIV/AIDS related morbidity and University of Port Harcourt, Choba, Rivers State, Nigeria 
mortality in developed countries has been dramatically reduced by the advent of effective combination of antiretroviral therapy (Emejulu et al., 2010). Antiretroviral therapies hamper the growth of the HIV virus, thereby causing the suppression of viral particle multiplication and eventually leading to decreased viral load, thereby prolonging the patient's life span. The impediments to effective use of Highly Active Antiretroviral Therapy (HAART) include the adverse effects associated with the use of these drugs. One of the adverse effects associated with highly active antiretroviral therapy is hepatotoxicity and is frequently reported in patients taking nevirapine containing highly active antiretroviral therapy. Case reports, clinical trials and other studies have linked nevirapine with hepatotoxicity in HIV patients taking nevirapine containing HAART.

Nevirapine is reported to be associated with early hypersensitivity reactions which can cause fulminant hepatitis leading to hepatic failure and death. It is also associated with late onset hepatotoxicity leading to liver enzymes elevation (Soriano et al., 2008). The World Health Organization (WHO) therefore recommends the use of nevirapine with caution and regular monitoring in patients who have baseline grade 1, 2 or 3 elevations of liver enzymes and $\mathrm{HBV}$ or $\mathrm{HCV}$ (WHO, 2006). In addition, nevirapine is not recommended in patients with a grade 4 elevation of liver enzymes. In January and March 2005, respectively, the US Food and Drug Administration (USFDA, 2005) and European Medicines Agency (EMEA) issued warnings in nevirapine package insert recommending against the initiation of nevirapine in adult women with CD4 count above 250 cells $/ \mathrm{mls}$ or in men with count above 400 cells $/ \mathrm{mls}$, because of a higher risk of hepatotoxicity. These data were derived mainly from a retrospective analysis of the Boehringer Ingelheim databases and further confirmed in a sub-analysis of the 2NN study (Stern et al., 2003; Vanleth et al., 2005). This research is in two parts. In this first part we reviewed reported(prevous and present) cases of nevirapine hepatotoxicity in animals and humans. We further analyzed reports on the relationship between the length of therapy, nevirapine plasma concentration and hepatotoxicity. The possible mechanisms of nevirapine induce hepatotoxicity were also evaluated. The second part which is on course is on the risk factors associated with hepatotoxicity caused by nevirapine containing HAART.

\subsection{Pharmacology of Nevirapine}

Nevirapine was the first dipyridozepinone Non Nucleoside Reverse Transcriptase Inhibitor (NNRTI) approved for use in HIV-infected patients. Its efficacy has been well demonstrated in numerous clinical trials. It has activity against HIV -1 but does not have significant activity against HIV-2 or other retroviruses (Harris and Montainer, 2000). It is easy to administer and is generally well absorbed. The pharmacokinetics of nevirapine is characterized by rapid and nearly completes oral absorption, rapid distribution and prolonged elimination (Cheeseman et al., 1993). Nevirapine bioavailability is $90 \%$ and is not impaired by food and antacids. It is approximately $60 \%$ protein bound and achieved cerebrospinal fluid levels that are $45 \%$ of those in plasma. Nevirapine has a volume of distribution of $1.21 \pm 0.09 \mathrm{~L} \mathrm{~kg}^{-1}$ (Smith et al., 2001; Podzamcer and Fumero, 2001). Nevirapine crosses the placenta and is reported to be secreted in breast milk. This has stimulated the use of this drug in the prevention of mother to child transmission of HIV (Mirochnick et al., 1998). Nevirapine mechanism of action involves binding directly to HIV-1 reverse transcriptase enzyme and causing a structural change that disrupts the formation of the active site and leads to impaired polymerization activity (Murphy and Montaner, 1996). Resistance leading to clinical treatment failure can occur with mutation at codon 100, 103, 106 and 108 of reverse transcriptase enzyme (Kuritkes, 2004; Eshleman et al., 2009). In vitro studies using human liver microsomes, demonstrated that CYP3A4 and CYP2B6 are primarily responsible for the oxidative metabolism of nevirapine to its major metabolites, although other CYP enzymes may have a secondary role (Erickson et al., 1999; Smith et al., 2001). Nevirapine metabolism is subject to auto induction of CYPs including CYPA4. This results in decrease in the terminal half-life from 45 $\mathrm{h}$ following a single dose to approximately $25-30 \mathrm{~h}$ after multiple dosing. The urinary excretion of nevirapine is minor, since only a small fraction $(<3 \%)$ is eliminated as parent compound in urine (Murphy and Montaner, 1996).

Nevirapine has been linked to some potentially serious adverse effects. One of these effects is nevirapine rash which is characterized by macular or popular eruptions commonly seen in the trunk, face and extremities. Pruritus is also associated with nevirapine. This stops when therapy is discontinued. Life threatening steven johnson's syndrome is rare but occurs (Johnson and Baraboutis, 2000). Severe and fatal hepatotoxicity has been reported with nevirapine use 
(Harris and Montaner, 2000; Dieterich et al., 2004). Other reported side effects of nevirapine include fatigue, headache, fever, somnolence and nausea. Clinically nevirapine is typically used as a component of highly active antiretroviral regimen. In addition nevirapine have been used in the prevention of mother to child transmission of HIV.

\subsection{Classification of Hepatotoxicity}

Liver toxicity is defined as an increase in aspartate Aminotransferase (AST) or alanine Aminotransferase (ALT) levels 5 times above the upper limit of normal (corresponding to WHO grade 3-4 toxicity). There have been discrepancy in the classification of hepatotoxicity. A standard toxicity grade scale is available and is used by AIDS research group. Patients with normal AST and ALT levels before treatment are classified with respect to changes relative to the upper limit of normal UNL. Hepatotoxicity is classified as grade $0(<1.25 \times \mathrm{ULN})$; grade $1(1.25-2.5 \times \mathrm{ULN})$; grade $2(2.6-5 \times \mathrm{ULN})$; grade 3 (5.1-10 $\times$ ULN) and grade $4(>5 \times \mathrm{ULN})$. Severe hepatotoxicity is defined as grade 3 and grade 4 changes in AST and/or ALT levels during highly active antiretroviral therapy (Kontorinis and Dieterich, 2003).

\subsection{Nevirapine Associated Hepatotoxicity}

Nevirapine associated hepatotoxicity has been a front burner especially in HIV patients taking nevirapine containing antiretroviral regimens. Reports have shown that there are two distinct types of nevirapine associated hepatotoxicity with characteristic features. The first type of nevirapine associated hepatotoxicity is an immune mediated hypersensitivity reaction which develops within 18 weeks of nevirapine therapy and it is said to be an early onset hepatotoxicity (AIDS INFO). The second type of nevirapine mediated hepatotoxicity occurs after 18 weeks of nevirapine therapy and it is said to be late onset and dose related (Mratinez et al., 2001; Clarke et al., 2000; Sulkowski et al., 2002). Furthermore, nevirapine hepatotoxicity is said to be associated with some risk factors which include, gender, CD4 cell count, co-infection with hepatitis B or $\mathrm{C}$ and pregnancy which will be reviewed in the second part of this work (Timmermans et al., 2005; Taiwo, 2006; Torti et al., 2007; Jamisse et al., 2007). In this review we were able to collect and analyze available information on case reports, clinical trials and other studies on nevirapine associated hepatotoxicity. Reported hepatotoxic effect of nevirapine in experimental animals was also captured in this work. The correlation between nevirapine plasma concentration and hepatotoxicity is discussed in the light of reported literature.

\subsection{Nevirapine Hepatotoxicity in Animals}

Animal models are used for experimental screening of pharmacological agents. Some pharmacological effects produced by drugs in experimental animals could reflect same or similar pharmacological effects in humans. This is Probably due to some similarities between humans and some animals. There are reports of nevirapine associated hepatotoxicity in experimental animal studies. In this aspect of our work we reviewed available information on nevirapine associated hepatotoxicity in experimental animals.

We will open this discussion with the work of Adaramoye et al. (2012) who investigated the toxic effects of 18 and $36 \mathrm{mg} \mathrm{kg}^{-1}$ of nevirapine in rats. Among other findings nevirapine was observed to increase the relative weight of the liver and elevated liver enzymes. Histopathological findings showed that nevirapine caused severe necrosis in the liver of the rats. Adewale et al. (2012) also evaluated the effects of coadministered lamivudine, nevirapine and zidovudine on hematological parameters and liver histology in female wistar rats. One of the outstanding findings is the hepatotoxic effect of this combination. This hepatotoxic effect was attributed to the presence of nevirapine in the administered regimen.

The adverse hepatic effect associated with the administration of nevirapine, lamivudine and stavudine was further studied using albino rats by Umar et al. (2008). In association with other findings they showed that both acute and sub-chronic administration of nevirapine resulted in significant increase in activities of aspartate aminotransferase and alanine amino transferase. They concluded that nevirapine could be associated with hepatotoxicity (Umar et al., 2008).

In a comparative toxicological evaluation of the hepatotoxic effects of some antiretroviral drugs on wistar albino rats, 200, 30 and $150 \mathrm{mg}$ of nevirapine, stavudine and lamivudine were administered respectively. Comparatively nevirapine produced the highest abnormality in the liver function of trated rats via abnormal elevation of liver enzymes (Sule et al., 2012). Comparatively some researchers have shown lack of disparity between nevirapine associated hepatotoxicity and other antiretrovirals (Bruck et al., 2008). In continuation of the evaluation of the hepatotoxic effect of nevirapine Kayode et al. (2011) evaluated the 
hematological and hepatic enzyme alterations associated with acute administration of some antiretroviral drugs. They showed that short term administration of SNP40 a drug containing stavudine, lamivudine and nevirapine to rats couldn't produce any significant change in the levels of aspartate aminotransferase and alanine aminotransferase when compared with the control. In the light of available literature, the hepatotoxic effect of nevirapine in animals is associated with elevation of liver enzymes with few incidences of liver necrosis.

\subsection{Hepatotoxicity of Nevirapine in Humans}

The use of nevirapine containing highly active antiretroviral therapy has tremendously reduced the progression of HIV disease. However hepatotoxicity is one of the limiting factors militating against the use of nevirapine containing highly active antiretroviral therapy in humans as reported in some clinical trials, cohort studies and case reports. In this part of the review we critically analyzed some of the reported studies carried out.

\subsection{Clinical and Cohort Studies}

We were able to collect reports from different quarters on nevirapine containing antiretroviral therapy associated with hepatotoxicity. One of these reports is the work of the Adult Aids Clinical Trial Group. They analyzed 21 studies involving 10611 patients and found that severe hepatotoxicity was observed in patients administered nevirapine containing antiretroviral drug and discontinuation of nevirapine was reported. Reister and colleagues further found that the rate of hepatotoxicity was $8.9 \%$ (95\% Confidence Interval (CI) 6.6 to $11.2 \%$ ) and $10.8 \%$ (95, CI 3.3 to $18.3 \%$ ), respectively, among patients receiving nevirapine and efavirenz. These two drugs were significantly more likely to be associated with grade 3 or 4 elevation of transaminases (Prakash et al., 2001). In a study involving 568 patients receiving NNRTI containing antiretroviral regimens, 312 and 256 patients received efavirenz and nevirapine respectively. Severe hepatotoxicity occurred throughout the duration of NNRTI therapy and was very common among patients taking nevirapine and co-infected with HBV (Sulkowski et al., 2002). This was further supported by the evaluation of Four hundred and sixty eight (468) subjects which were enrolled in a study between August 1999 and February 2000. Two hundred and thirty four (234) subjects were randomized to receive lamivudine. Eighty-two percent of subjects (385/468) were enrolled in the nevirapine group. The frequency of hepatotoxicity was $17 \%(66 / 385)$ in the nevirapine group and $0 \%(0 / 83)$ in the efavirenz group.

To investigate the incidence of hepatotoxicity in Acquired Immune Deficiency Syndrome (AIDS) patients taking nevirapine containing HAART, 330 AIDS patients were enrolled in this retrospective study. Two hundred and sixty seven (267) out of 330 patients received nevirapine based highly antiretroviral therapy while 63 cases received efavirenz based highly active antiretroviral therapy. It was observed that 133 out of $267(49.8 \%)$ patients on nevirapine based highly active antiretroviral therapy had at least one episode of alanine aminotransferase elevation. Conclusively it was reported that nevirapine related hepatotoxicity was common among naive HIV infected patients in this cohort study (Gao et al., 2010).

Furthermore Chu et al. (2010) conducted a study involving 1809 HIV-infected naive adults initiating nevirapine-based antiretroviral therapy. The primary outcome of this study was early hepatotoxicity while the secondary outcome was hepatotoxicity and mortality at six months. In a study involving $930 \mathrm{HIV}$-infected patients who were placed on nevirapine between 1998 and 2003, it was reported that the rate of severe hepatotoxicity due to nevirapine was high (Jonckheere et al., 2012). This was supported by a comprehensive analysis of 17 randomized clinical trials of nevirapine treated patients; results showed that $10 \%$ of all nevirapine treated patients had elevated levels of alanine aminotransferase and aspartate aminotransferase enzymes greater than 5 times the ULN. Almost two-third $(6.3 \%)$ of nevirapine treated patients with elevated alanine aminotransferase or aspartate aminotransferase enzymes were asymptomatic. Symptomatic hepatic events were seen in 4.9\% (3.2-8.9\%) of nevirapine treated patients (Dieterich et al., 2004).

Martinez et al. (2001), in a prospective cohort study evaluated 610 patients who were prescribed nevirapinecontaining antiretroviral regimens. They reported that hepatotoxicity was common in HVI-1-infected patients receiving nevirapine-containing regimens and the incidence steadily increased overtime.

A study of 540 HIV adult patients who were monitored consecutively taking (d4T/3TC/NVP) Fixed-Dose Combinations (FDC) was performed by Meysonnier and colleagues. Pregnant woman, hepatitis B and C patients were excluded. Among 497 patients with at least two alanine aminotransferase tests, 78 episodes of hepatotoxicity $(15.7 \%)$ including six cases of grade 3 hepatotoxicity $(1.2 \%)$ and grade 4 hepatotoxicity or symptomatic hepatotoxicity were reported (Meyssonnier et al., 2008). 
Five hundred and eighty two (582) patients receiving nevirapine as a component of highly active antiretroviral therapy containing $(72 \%$ males) were used in this study. Among other parameters evaluated, $10.2 \%$ of treated patients manifested a grade 3 or greater increase in aminotransferase levels reflecting an overall incidence rate equal to 5.3 cases/100 person/years. This led to treatment discontinuation in $3.9 \%$ of the cases. Conclusively they reported that the risk of nevirapine containing antiretroviral regimens associated hepatotoxicity was low. They advised that it should be used with caution in patients with hepatitis and elevated liver enzymes (Magiolo et al., 2007). In the light of the above reports, there is evidence to show that nevirapine could be associated with hepatotoxicity. The rate of mortality with respect to nevirapine associated hepatotoxicity was quite low only limited cases were observed. The rate of discontinuation of nevirapine due to nevirapine associated hepatotoxicity was also reported and this may not hinder the clinical use of nevirapine except when risk outweighs benefit.

\subsection{Case Report}

Off recent there are increasing case reports on hepatotoxicity associated with nevirapine during therapy in humans. We systematically collected and analyzed some of these reports. One of these case reports was documented by Buyse et al. (2006). He and co researchers reported a case of liver transplant due to nevirapine associated fulminant hepatitis; laboratory evaluation revealed elevated levels of alanine amino transferase, aspartate amino transferase, gamma glutamyl transpeptidase and total bilirubin. Liver biopsy showed massive necrosis in the centrilobular and mediolobular areas, coagulative necrosis was associated with inflammatory reaction. Bera et al. (2012) also reported two cases of maternal death following therapy with nevirapine containing antiretroviral regimen. In these patients, liver function was observed to be abnormal due to elevation in liver function parameters. Hepatotoxicity associated with nevirapine was also reported by Baichi (2003). He reported a case of nevirapine induced hepatitis in a $48 \mathrm{yr}$ old male. This was characterized by hepatomegaly and abnormal liver function which was associated with abnormal elevation of liver enzymes. Similar observation was also reported (Reiter, 1997; Bundow et al., 2001).

The above case report was supported by the work of Pillero and Bonnie who documented a case of hepatotoxicity suspected to be induced by nevirapine.
Laboratory evaluation showed abnormal elevation in liver enzymes; similar observations were reported by some scholars (Mens and Katzenstein, 2005). Nevirapine has also been shown to be associated with hepatic failure as observed by Cattelan et al. (1999). This case report was associated with jaundice and hepatomegaly; laboratory evaluations disclosed abnormal elevation of liver enzymes. Ultrasonography disclosed enlargement of the liver with a non homogenous echo texture and abundant ascites.

Furthermore, therapy with nevirapine containing antiretroviral regimen was reported to be associated with immunoallergic hepatitis. Like most case reports it was also associated with abnormal elevations of liver enzymes and jaundice (Knudtson et al., 2003). Gokengin and Yamazahan (2002) reported two cases of life threatening hepatoxicity in health workers who were administered nevirapine for post exposure prophylaxis. One of the cases required liver transplant after developing fulminant hepatitis and end stage hepatic failure (Johnson and Baraboutis, 2000). From March 1997-September 2000 FDA received 22 cases of serious adverse events related to nevirapine taken for post exposure prophylaxis. These 22 events include 12 cases of hepatotoxicity (CDC, 2001).

Patel et al. (2004) described 30 cases of hepatoxicity including 14 severe cases and one case of fulminant hepatic necrosis associated with nevirapine post exposure prophylaxis; although they concluded that the presence of other antiretrovirals must have augmented the effect of nevirapine associated hepatotoxicity. Manfred (2001), also reported two cases of health care workers with possible exposure to human immunodeficiency virus who developed adverse reactions after receiving multiple doses of nevirapine/zidovudine/lamivudine as Post Exposure Prophylaxis (PEP). One of the patients developed fulminant hepatic failure resulting to liver transplant.

Maniar et al. (2006) published a case of fulminant hepatitis associated with nevirapine containing regimens zidovudine/lamivudine/nevirapine (AZT/3TC/NVP). Abdominal ultrasound revealed hepatomegaly, fatty infiltration, gall bladder wall thickening, bulky pancreas and moderate ascites. A case of severe leucopenia associated with mild hepatotoxicity in a HIV carrier treated with nevirapine was reported by Shahar et al. (2004). This was characterized by abnormal elevation of liver enzymes. Initially this patient was placed on zidovudine/lamivudine/indinnavir. Because of mild symptoms of hypo dystrophy the indinavir was replaced with nevirapine. 


\subsection{Nevirapine Concentration and Hepatoxicity}

The pharmacologogy of nevirapine makes it an attractive candidate for therapeutic drug monitoring, as previous studies have indicated a significant relationship between nevirapine trough concentrations and virologic response (Veldkamp et al., 2001; Vries-Sluijs et al., 2003). Also, high concentrations were linked to increase risk of liver toxicity and hepatic injury in patients with chronic hepatitis C (Dailly et al., 2004; Nunez et al., 2003; Kappelhoff et al., 2005). Inter-individual variability of nevirapine plasma concentrations among HIV infected adults was common in routine clinical practice (50\%). The variability could be partially explained by the differences in ethnicity, gender, polymorphisms in enzyme, transporter genes, hepatitis and concomitant medications (Haas, 2005; Kappelhoff et al., 2005; Ma et al., 2005; Stohr et al., 2008).

Requena et al. (2002) investigated the effect of nevirapine plasma concentration on liver enzyme elevations and observed that among patients with chronic hepatitis $\mathrm{C}$ coinfection, nevirapine concentrations $>6 \mathrm{mg} \mathrm{mL}^{-1}$ were associated with $92 \%$ risk of liver toxicity. Therefore, monitoring nevirapine levels, especially in individuals with chronic hepatitis $\mathrm{C}$ co infection, might be warranted. However, a prospective population pharmacokinetic study and the 2NN study suggested no correlation between nevirapine trough concentrations and liver enzyme levels (Dailly et al., 2004; Kappelhoff et al., 2005).

Furthermore, in a research to establish a relationship between nevirapine plasma concentration and hepatotoxicity, 227 HIV-infected patients taking nevirapine were evaluated. Their Blood samples were taken $12 \mathrm{hr}$ post dose and $24 \mathrm{~h}$ post dose for measurement of nevirapine concentration six months after treatment initiation. Result showed that nevirapine hepatoxicity was associated with median nevirapine trough concentration among male patients and hepatitis $\mathrm{C}$ virus co-infection. Among the 227 patients screened 191 patients were qualified for the liver toxicity analysis. The incident of liver toxicity was 71\% (136/191) including 43 patients $(23 \%)$ with severe liver toxicity within 12 weeks of starting therapy. The correlation between nevirapine concentration, efficiency and hepatotoxicity suggested the benefit of dosage adjustment based on therapeutic drug monitoring (Wang et al., 2011). A study to assess the differences in the pharmacokinetics of nevirapine between patients with $\mathrm{HIV} / \mathrm{HCV}$ confection and HIV infection that could explain higher rates of hepatotoxicity was performed. In this study 18 patients; with seven (7) HIV/HCV confected and eleven (11) HIV non- infected were used. Observation in this study highlights the possibility of accumulating drug levels under nevirapine therapy and thus pharmacokinetics measurement in individual cases with expected nevirapine-associated hepatotoxicity may be warranted (Vogel et al., 2009). This agreed with an evaluation of toxicity data for 8216 patients treated with nevirapine containing antiretroviral regimens which showed that duration of nevirapine therapy may influence the outcome of nevirapine associated hepatotoxicity. Results also showed that increased duration of nevirapine therapy could predict nevirapine hepatotoxicity (McKoy et al., 2009).

Maat et al. (2002; 2003) evaluated174 individuals infected with HIV and observed that the plasma concentration of nevirapine does not appear to be a risk factor in nevirapine associated hepatotoxicity. A prospective pharmacokinetic study of nevirapine was performed to test the relationship between hepatotoxicity and nevirapine trough plasma concentration and to identity which covariate could influence nevirapine pharmacokinetics. In this prospective study seventyseven (77) HIV infected patients were used. It was observed that nevirapine trough plasma concentration has no correlation with hepatotoxicity (Dailly et al., 2004). This is in resonance with an investigation of the relationship between total and unbound plasma concentration of nevirapine and liver enzymes (Alanine amino transferase and Gama-glutaryl transferase). A total of eighty-five (85) HIV infected patients taking nevirapine containing antiretroviral were used; result showed that there was no strong association between nevirapine concentration and hepatotoxicity (Almond et al., 2004)

Dong et al. (2012) evaluated the relationship between nevirapine pharmacokinetics and rash/hepatotoxicity in three hundred and fifty nine (359) women starting antiretrovirals. Among other observations it was reported that hepatotoxicity is not associated with higher nevirapine exposure. There are opposing views with respect to the information collected on the effect of nevirapine plasma concentration and nevirapine associated hepatotoxicity. This leaves more room for further evaluation.

\subsection{Mechanisms of Nevirapine Hepatotoxicity}

The mechanism of nevirapine induced hepatotoxicity is not well understood but it is known that drug induce 
hepatotoxicity can occur through different mechanisms (Lee, 2003). Some of the possible mechanisms of drug induce hepatotoxicity include direct toxicity, mitochondria damage and hypersensitivity reaction (Grattagliano et al., 2002; Dykens and Will, 2007).

\subsection{Direct Toxicity}

Nevirapine and some drugs are known to be metabolized by the liver hence could induce direct toxicity on the liver. Drugs metabolized by the liver through the Cytochrome pathway may cause liver toxicity when there are polymorphisms in the enzymes (Bissell et al., 2001). The most important drug metabolizing enzyme for the creation of hepatotoxic reactive metabolites is the polymorphic Cytochrome P450 (CYP450) family that mediates oxidative phase-I drug metabolism. However, conjugative phase-II metabolism may also result in hepatotoxic metabolites, e.g., acyl glucuronides are well known to cause DILI (Spahn-Langguth and Benet, 1992; Boelsterli, 2002). Reactive metabolites can exert initial cell stress through a wide range of mechanisms including depletion of glutathione $(\mathrm{GSH})$, or binding to enzymes, lipids, nucleic acids and other cell structures. Furthermore reactive metabolites or parent drugs may specifically inhibit other hepatocellular functions (Pauli-Magnus et al., 2005). Since many of the antiretroviral drugs are metabolized by liver enzymes polymorphism in the enzymatic complexes might lead to heterogenicity in drug metabolism. This can lead to the development of hepatotoxicity in some individuals. Some drugs might stimulate or activate stress pathway leading to hepatic stress which could be observed in nevirapine (Leist et al., 1998).

\subsection{Hypersensitivity Reaction}

Hypersensitivity reaction is an idiosyncratic reaction exhibited by the host with respect to an administered drug. Allergic idiosyncratic hepatotoxicity is characterized by the presence of typical symptoms and signs of adaptive immune reactions, including fever, skin reactions, eosinophilia, formation of autoantibodies and a short latency time particularly after re-exposure (Russmann et al., 2009). Hypersensitivity could be immune mediated. Mediated immune drug reaction could be associated with the generation of neoantigens produced by the reaction of liver proteins with reactive drug metabolites. Several authors have linked nevirapine with hypersensitivity reactions (Martin et al., 2005; Pilliero and Purdy, 2001). Nevirapine hypersensitivity is said to develop in the first few weeks of therapy and is characterized by rash, fever, hypotension along with hepatic dysfunction (Johnson and Baraboutis 2000).

\subsection{Mitochondria Toxicity}

Drugs (or their metabolites) can induce mitochondrial dysfunction in hepatocytes through several mechanisms. One of these mechanisms is the induction of MPT. Several drugs can trigger MPT in liver mitochondria, leading to apoptosis and/or necrosis. These drugs cause 'cytolytic' hepatitis, leading occasionally to fulminant hepatic failure. Some drugs (or metabolites) can directly trigger MPT in isolated mitochondria (Pessayre et al., 2007; Trost and Lemasters, 1996; Masubuchi et al., 2006). The molecular mechanisms whereby these drugs induce MPT are often poorly understood. FAO impairment can also lead to mitochondrial dysfunction in hepatocytes. Drugs can also impair the mitochondrial oxidation of fatty acids, leading to their accretion as triglycerides in the cytoplasm of the hepatocytes. Although free fatty acids can also accumulate, this seems to happen preferentially when FAO is severely inhibited (Fromenty and Pessayre, 1995). FAO can be impaired in different ways. Some drugs directly impair mitochondrial FAO by inhibiting FAO enzymes (Deschamps et al., 1991; Pessayre et al., 1999; 2007).

Furthermore NRTIs are known to induce mitochondria damage. Human mitochondrial DNA polymerase is inhibited by NRTIs (Kontorinis and Dieterich, 2003). Depletion of mitochondrial DNA impairs the cellular respiration chain and inhibits pyruvate and folic acid pathways. Mitochondrion appears enlarged and swollen with metrical densities and occasional vacuoles (Moyle, 2000). In severe NRTIs mitochondria toxicity, macrovasicular hepatic cholestasis, giant mitochondrial and intrahepatic cholestatic will be observed (Moyle, 2000; Spengler et al., 2002).

\section{CONCLUSSION}

In this review it is discovered that nevirapine might be hepatotoxic. Liver function status of HIV patients eligible for nevirapine should be evaluated. Expecially in patients with HIV/Hepatitis coinfection. Also coadministration of nevirapine with other drugs that have adverse hepatotoxic effect or could potentiate the hepatotoxic effect of nevirapine should be avoided or use with caution. 


\section{REFERENCES}

Adaramoye, O.A., O.A. Adesanoye, O.M. Adewumi and O. Akanni, 2012. Studies on the toxicological effect of nevirapine, an antiretroviral drug, on the liver, kidney and testis of male Wistar rats. Hum. Exp. Tox., 13: 676-685. DOI: $10.1177 / 0960327111424304$

Adewale, O.A., O.O. Jubril, H.I. Olwasegun, H.A. Selinot and A.R. Ayoola, 2012. Effects of a single pill 3-drug combination of lamivudine, nevirapine and zidovudine on blood parameters and liver histology in female wistar rats. Am. J. Med. Med. Sci., 2: 71-74. DOI: 10.5923/j.ajmms.20120204.02

Almond, L.M., M. Boffito, P.G. Hoggard, S. Bonora and R. Raiteri et al., 2004. The relationship between nevirapine plasma concentrations and abnormal liver function tests. AIDS Res. Hum. Retroviruses, 20: 716-722. PMID: 15307917

Baichi, M., 2003. Nevirapine hepatotoxicity: Case report and discussion. Med. Forum.

Bera, E., D. Naidoo and M. Williams, 2012. Maternal deaths following nevirapine-based antiretroviral therapy. S. Afr. J. HIV Med., 13: 196-197. DOI: 10.7196/SAJHIVMED.869

Bissell, D.M., G.J. Gores, D.J. Laskin and J.H. Hoofnagle, 2001. Drug-induced liver injury: Mechanisms and test systems. Hepatology, 33: 1009-1013. PMID: 11283870

Boelsterli, U.A., 2002. Mechanisms of NSAID-induced hepatotoxicity: Focus on nimesulide. Drug. Saf., 25: 633-648. PMID: 12137558

Bruck, S., S. Witte, J. Brust, D. Schuster and F. Mosthaf et al., 2008. Hepatotoxicity in patients prescribed efavirenz or nevirapine. Eur. J. Med. Res., 13: 343-348. PMID: 18700192

Bundow, D., L. Rosoff and D.M. Abonlafia, 2001. Optimal treatment of nevirapine-associated hepatotoxicity remains uncertain. AID Read., 11: 577-580. PMID: 11789021

Buyse, S., E. Vibert, M. Sebagh, T. Antonini and P. Ichei et al., 2006. Liver transplantation for fulminant hepatitis related to nevirapine therapy. Liver Transpl., 12: 1880-1882. PMID: 17133571

Cattelan, A.M., E. Erne, A. Salatin, M. Trenvezoli and G. Carretta, 1999. Severe hepatic failure related to nevirapine treatment. Clin. Infect. Dis., 29: 455-456. PMID: 10476768
CDC, 2001. Serious adverse events attributed to nevirapine regimens for postexposure prophylaxis after HIV exposures-Worldwide, 1997-2000. MMRN., 49: 1153-1156.

Cheeseman, S.H., S.E. Hattox, M.M. McLaughlin, R.A. Koup and C. Andrews et al., 1993. Pharmacokinetics of nevirapine: Initial single-risingdose study in humans. Antimicrob. Agents Chemother., 37: 178-182. PMID: 8452345

Chu, K.M., A.M. Boulle, N. Ford, E. Goemaere and V. Asselman et al., 2010. Nevirapine-associated early hepatotoxicity: Incidence, risk factors and associated mortality in a primary care ART programme in South Africa. PLoS One, 5: 9183-9183. DOI: 10.1371/journal.pone.0009183

Clarke, S., P. Harrington, C. Condon, D. Kelleher and O.P. Smith et al., 2000. Late onset hepatitis and prolonged deterioration in hepatic function associated with nevirapine therapy. Int. J. STD AIDS, 11: 336-337. PMID: 10824944

Dailly, E., E. Billaud, V. Reliquet, S. Breurec and P. Perre et al., 2004. No relationship between high nevirapine plasma concentration and hepatotoxicity in HIV-1-infected patients naive of antiretroviral treatment or switched from protease inhibitors. Eur. J. Clin. Pharmacol., 60: 343-348. PMID: 15156302

Deschamps, D., C. Fisch, B. Fromenty, A. Berson and C. Degott et al., 1991. Inhibition by salicylic acid of the activation and thus oxidation of long chain fatty acids. Possible role in the development of Reye's syndrome. J. Pharmacol. Exp. Ther., 259: 894-904. PMID: 1941634

Dieterich, T.D., P.A. Robison, J. Love and J.O. Stern, 2004. Drug-induced liver injury associated with the use of nonnucleoside reverse-transcriptase inhibitors. Clin. Infect. Dis., 38: 508-509. PMID: 14986279

Dong, B.J., Y. Zheng, M.D. Hughes, A. Frymoyer and D. Verotta et al., 2012. Nevirapine pharmacokinetics and risk of rash and hepatitis among HIV-infected sub-Saharan African women. AIDS, 26: 833-841. DOI: 10.1097/QAD.0b013e328351a521

Dykens, J.A. and Y. Will, 2007. The significance of mitochondrial toxicity testing in drug development. Drug. Discov. Today, 12: 777-785. DOI: 10.1016/j.drudis.2007.07.013

Emejulu A.A., C. OUjowundu, C.U. Igwe and V.A. Ouwuliri, 2010. Hepatotoxicity of antiretroviral drugs in HIV seropositive Nigerian patients Aust. J. Basic Applied Sci., 4: 4275-4278. 
Erickson, D.A., G. Mather, W.F. Trager, R.H. Levy and J.J. Keirns, 1999. Characterization of the in vitro biotransformation of the HIV-1 reverse transcriptase inhibitor nevirapine by human hepatic cytochromes P-450. Drug. Metab. Dispos., 27: 1488-1495. PMID: 10570031

Eshleman, S.H., L.A. Guay, A. Mwatha, S.P. Cunningham and E.R. Brown et al., 2009. Comparison of Nevirapine (NVP) resistance in Ugandan women 7 days vs. 6-8 weeks after singledose nvp prophylaxis: HIVNET 012. AIDS Res. Retroviruses, 20: 595-599. PMID: 15242535

Fromenty, B. and D. Pessayre, 1995. Inhibition of mitochondrial beta-oxidation as a mechanism of hepatotoxicity. Pharmacol. Ther., 67: 101-154. PMID: 7494860

Gao, S.E., X.E. Gui, L.P. Deng and Y.X. Zhang, 2010. Nevirapine related hepatotoxicity: The prevalence and risk factors in a cohort of ART naive Han Chinese with AIDS. Zhonghua. Gan. Zang. Bing. Za. Zhi., 18: 689-693. DOI: 10.3760/cma.j.issn.1007-3418.2010.09.011

Gokengin, D and T. Yamazahan, 2002. Hepatic adverse events during highly active antiretroviral therapy containing nevirapine: A case report. Ann. Clin. Microbiol. Antimicrob., 1: 1-1. DOI: 10.1186/14760711-1-1

Grattagliano, I., P. Portincasa, V.O. Palmieri and G. Palasciano, 2002. Overview on the mechanisms of drug-induced liver cell death. Ann. Hepatol., 1: 162168. PMID: 15280801

Haas, D.W., 2005. Pharmacogenomics and HIV therapeutics. J. Infect. Dis., 191: 1397-1400. PMID: 15809895

Harris, M. and J.S. Montaner, 2000. Clinical uses of nonnucleoside reverse transcriptase inhibitors. Rev. Med. Virol., 10: 217-229. PMID: 10891870

Jamisse, L., J. Balkus, J. Hitti, S. Gloyd and R. Manuel et al., 2007. Antiretroviral-associated toxicity among HIV-1-seropositive pregnant women in Mozambique receiving nevirapine-based regimens. J. Acquir. Immune Defic. Syndr., 44: 371-376. PMID: 17259905

Johnson, S. and J.G. Baraboutis, 2000. Adverse effects associated with use of nevirapine in HIV postexposure prophylaxis for 2 health care workers. JAMA, 284: 2722-2723. PMID: 11105175

Jonckheere, S., J. Yombi, L. Belcher and A. Vicent, 2012. Nevirapine-associated liver toxicity and hypersensitivity reactions in a cohort of HIV-1infected patients,clinical analysis. Retrovirology, 1: 10-10. DOI: 10.1186/1742-4690-9-S1-P10
Kappelhoff, B.S., F.V. Leth, P.A. Robinson, T.R. MacGregor and E. Baraldi et al., 2005. Are adverse events of nevirapine and efavirenz related to plasma concentrations? Antivir. Ther., 10: 489-498. PMID: 16038474

Kayode, A.A., T.O. Kayode, O.A. Areyeun and M.C. Stephen, 2011. Hematological and hepatic enzyme alterations associated with acute administration of antiretroviral drugs. Asian J. Pharmacol. Toxicol., 6: 293-302. DOI: 10.3923/pt.2011.293.203

Knudtson, E., M. Para, H. Boswell and P. Fan-Havard, 2003. Drug rash with eosinophilia and systemic symptoms syndrome and renal toxicity with a nevirapine-containing regimen in a pregnant patient with human immunodeficiency virus. Obstet. Gynecol., 101: 1094-1097. PMID: 12738113

Kontorinis, N. and D. Dieterich, 2003. Hepatotoxicity of antiretroviral therapy. AIDS Rev., 5: 36-43. PMID: 12875106

Kuritkes, D.R., 2004. Preventing and managing antiretroviral drug resistance. AIDS Patients Care STDS, 18: 259-273. PMID: 15186710

Lee, W.M., 2003. Drug-induced hepatotoxicity. N. Engl. J. Med., 349: 474-485. DOI: 10.1056/NEJMra021844

Leist, M., F. Gantner, G. Kunstle and A. Wendel, 1998. Cytokine-mediated hepatic apoptosis. Rev. Physiol. Biochem. Pharmacol., 133: 109-155. PMID: 9600012

Ma, Q., O.O. Okusanya, P.F. Smith, R. Dicenzo and J.C. Slish et al., 2005. Pharmacokinetic drug interactions with non-nucleoside reverse transcriptase inhibitors. Expert. Opin. Drug. Metab. Toxicol., 1: 473-485. PMID: 16863456

Maat, M.M.D., R.A. Mathot, A.I. Veldkamp, A.D. Huitma and J.W. Mulder et al., 2002. Hepatotoxicity following nevirapine-containing regimens in HIV-1infected individuals. Pharmacol. Res., 46: 295-300. PMID: 12220974

Maat, M.M.D., R.T. Heine, E.C.V. Gorp, J.W. Mulder and A.T. Mairuhu et al., 2003. Case series of acute hepatitis in a non-selected group of HIV-infected patients on nevirapine-containing antiretroviral treatment. AIDS, 17: 2209-2214. PMID: 14523278

Magiolo, F., C. Arici, M. Alriodi, D. Ripamonti and G. Quinzan et al., 2007. Reasons for discontinuation of nevirapine-containing HAART: Results from an unselected population of a large clinical cohort. J. Antimicrob. Chemother., 59: 569-572. PMID: 17255141 
Manfred, H., 2001. Nevirapine and postexposure prophylaxis for human immunodeficiency virus. JAMA, 285: 883-883. DOI: 10.1001/jama.285.7.879

Maniar, J.K., S.R. Shah, R. Verma, R. Kamath and P. Gupta et al., 2006. Nevirapine-induced fulminant hepatitis. J. Assoc. Physicians. Ind., 54: 957-958. PMID: 17334017

Martin, A.M., D. Nolan, I. James, P. Cameron and J. Keller et al., 2005. Predisposition to nevirapine hypersensitivity associated with HLA-DRB1*0101 and abrogated by low CD4 T-cell counts. AIDS, 19: 97-99. PMID: 15627041

Masubuchi, Y., S. Kano and T. Horie, 2006. Mitochondrial permeability transition as a potential determinant of hepatotoxicity of antidiabetic thiazolidinediones. Toxicology, 222: 233-239. PMID: 16621215

McKoy, J.M., C.L. Bennett, M.H. Scheetz, V. Differding and K.L. Kimberly et al., 2009. Hepatotoxicity associated with long-versus short-course HIVprophylactic nevirapine use: A systematic review and meta-analysis from the Research on Adverse Drug events And Reports (RADAR) project. Drug Saf., 32: 147-158. PMID: 19236121, DOI: 10.2165/00002018-200932020-00007

Mens, H. and T.L. Katzenstein, 2005. Hepatic failure due to nevirapine treatment for HIV Infection. Ugeskr Laeger., 167: 4359-4359. PMID: 16287519

Meyssonnier, V., D. Costagliola and P.E. Caumes, 2008. Nevirapine-associated toxicity in Niger. HIV Med., 9: 62-63. DOI: 10.1111/j.1468-1293.2008.00516.x

Mirochnick, M., T. Fenton, P. Gagnier, J. Pav and M. Gwynne et al., 1998. Pharmacokinetics of nevirapine in human immunodeficiency virus type 1-infected pregnant women and their neonates. Pediatric AIDS clinical trials group protocol 250 Team. J. Infect. Dis., 178: 368-374. PMID: 9697716

Moyle, G., 2000. Toxicity of antiretroviral nucleoside and nucleotide analogues: Is mitochondrial toxicity the only mechanism? Drug. Saf., 23: 467-781. PMID: 11144657

Martinez, E., J.L. Blanco, J.A. Arnaiz, J.B. Perez-Cuevas and A. Mocroft et al., 2001. Hepatotoxicity in HIV1 -infected patients receiving nevirapine-containing antiretroviral therapy. AIDS, 15: 1261-1268. PMID: 11426070

Murphy, R. and J. Montaner, 1996. Drug evaluations anti-infectives: Nevirapine: A review of its development, pharmacological profile and potential for clinical use. Exp. Opin. Invest Drug., 5: 11831199. DOI: $10.1517 / 13543784.5 .9 .1183$
Nunez, M., D. Gonzalez-Requena, J. Gonzalez-Lahoz and V. Soriano, 2003. Short communication: Interactions between nevirapine plasma levels, chronic hepatitis $\mathrm{C}$ and the development of liver toxicity in HIV-infected patients. AIDS Res. Hum. Retroviruses, 19: 187-188. PMID: 12689410

Patel, S.M., S. Johnson, S.M. Belknap, J. Chan and B.E. Sha et al., 2004. Serious adverse cutaneous and hepatic toxicities associated with nevirapine use by non-HIV-infected individuals. J. Acquir. Immune Defic. Syndr., 35: 120-125. PMID: 14722442

Pauli-Magnus, C., B. Stieger, Y. Meier, G.A. KullakUblick and P.J. Meier, 2005. Enterohepatic transport of bile salts and genetics of cholestasis. J. Hepatol., 43: 342-357. PMID: 15975683

Pessayre, D., A. Mansouri, D. Haouzi and B. Fromenty, 1999. Hepatotoxicity due to mitochondrial dysfunction. Cell Biol. Toxicol., 15: 367-373. PMID: 10811531

Pessayre, D., B. Fromenty, A. Mansouri and A. Berson, 2007. Hepato Toxicity Due to Mitochondrial Injury. In: Drug-Induced Liver Disease, Kaplowitz, N. and L.D. DeLeve (Eds.), Inform Healthcare, New York, ISBN-10: 0849398967, pp: 49-84.

Pilliero, P.J. and B. Purdy, 2001. Nevirapine-induced hepatitis: A case series and review of the literature. AIDS Read., 11: 379-382. PMID: 11494710

Podzamcer, D. and E. Fumero, 2001. The role of nevirapine in the treatment of HIV-1 disease. Exp. Opin. Pharmacother., 2: 2065-2078. PMID: 11825335

Prakash, M., V. Poreddy, L. Tiyyagura and M. Bonacini, 2001. Jaundice and hepatocellular damage associated with nevirapine therapy. Am. J. Gastroenterol., 96: 1571-1573. PMID: 11374701

Reiter, G.S., 1997. Hepatitis in an HIV-infected man. AIDS Clin. Care, 9: 78-81. PMID: 11364758

Requena, D.G.D., M. Nunez, I. Jimenez-Nacher and V. Soriano, 2002. Liver toxicity caused by nevirapine. AIDS, 16: 290-291. PMID: 11807315

Russmann, S., G.A. Kullak-Ublick and I. Grattagliano, 2009. Current concepts of mechanisms in druginduced hepatotoxicity. Curr. Med. Chem., 16: 3041-3053. PMID: 19689281

Shahar, E., N. Krivoy, S. Weltfriend and S. Pollach, 2004. Severe leukopenia associated with mild hepatotoxicity in an HIV carrier treated with nevirapine. J. Inf. Dis., 57: 212-213. PMID: 15507779 
Smith, P.F., R. DiCenzo and G.D. Morse, 2001. Clinical pharmacokinetics of non-nucleoside reverse transcriptase inhibitors. Clin. Pharmacokinet., 40: 893-905. PMID: 11735608

Soriano, V., M. Puoti, P. Garcia-Gasco, K.J. Rockstroh and Y. Benhamou et al., 2008. Antiretroviral drugs and liver injury. AIDS, 22: 1-13. PMID: 18090386

Spahn-Langguth, H. and L.Z. Benet, 1992. Acyl glucuronides revisited: Is the glucuronidation process a toxification as well as a detoxification mechanism? Drug. Metab. Rev., 24: 5-47. PMID: 1555494

Spengler, U., M. Lichterfeld and J.K. Rockstroh, 2002. Antiretroviral drug toxicity--a challenge for the hepatologist? J. Hepatol., 36: 283-294. PMID: 11830343

Stern, J.O., P.A. Robinson, J. Love, S. Lanes and M.S. Imperiale et al., 2003. A comprehensive hepatic safety analysis of nevirapine in different populations of HIV infected patients. J. Acquir. Immune Defic. Syndr., 34: 21-33. PMID: 14562855

Stohr, W., D. Back, D. Dunn, C. Sabin and A. Winston et al., 2008. Factors influencing efavirenz and nevirapine plasma concentration: Effect of ethnicity, weight and co-medication. Antivir. Ther., 13: 675685. PMID: 18771051

Sule, O.J., Godwin, J. and A.I. Nnopu, 2012. Biochemical investigation of hepatotoxic effects of antiretroviral drugs on wistar Albino rats. J. Phys. Pharm. Adv., 2: 171-175.

Sulkowski, M.S., D.L. Thomas, S.H. Mehta, R.E. Chaisson and R.D. Moore, 2002. Hepatotoxicity associated with nevirapine or efavirenz-containing antiretroviral therapy: Role of hepatitis C and B infections. Hepatology, 35: 182-189. PMID: 11786975

Taiwo, B.O., 2006. Nevirapine toxicity. Int. J. STD AIDS, 17: 364-369. PMID: 16734954

Timmermans, S., C. Tempelman, M.H. Godfried, J. Nellen and J. Dieleman et al., 2005. Nelfinavir and nevirapine side effects during pregnancy. AIDS, 19: 795-799. PMID: 15867493

Torti, C., S. Costarelli, A.D. Silvestri, E. Quiros-Roldan and G. Lapadula et al., 2007. Analysis of severe hepatic events associated with nevirapine-containing regimens: CD4+ T-cell count and gender in hepatitis $\mathrm{C}$ seropositive and seronegative patients. Drug. Saf., 30: 1161-1169. PMID: 18035868
Trost, L.C. and J.J. Lemasters, 1996. The mitochondrial permeability transition: A new pathophysiological mechanism for Reye's syndrome and toxic liver injury. J. Pharmacol. Exp. Ther., 278: 1000-1005. PMID: 8819478

Umar, R.A., S.W. Hassan, M.J. Ladan and I.K. Matazu and B. Shehu, 2008. Adverse hepatic effects associated with administration of antiretroviral drugs (nevirapine, lamivudine and stavudine) to albino rats: Implication for management of patients with HIV/AIDS. Asian J. Biochem., 3: 19-25. USFDA, 2005. Center for drug evaluation and research. U.S. Food and Drug Administration.

Vanleth, F., S. Andrews, B. Grinsztejn, E. Wilkins and M.K. Lazanas et al., 2005. The effect of baseline CD4 cell count and HIV-1 viral load on the efficacy and safety of nevirapine or efavirenz-based first-line HAART. AIDS, 19: 463-471. PMID: 15764851

Veldkamp, A.I., G.J. Weverling, J.M. Lange, J.S. Montaner and P. Reiss et al., 2001. High exposure to nevirapine in plasma is associated with an improved virological response in HIV-1-infected individuals. AIDS, 15: 1089-1095. PMID: 11416710

Vogel, M., N. Bertram, J.C. Wasmuth, C. Wyen and E. Voigt et al., 2009. Nevirapine pharmacokinetics in $\mathrm{HIV}$-infected and HIV/HCV-coinfected individuals. J. Antimicrob. Chemother., 63: 988-991. PMID: 19270314

Vries-Sluijs, T.E.D., J.P. Dieleman, D. Arts, A.D. Huitema and J.H. Beijnen et al., 2003. Low nevirapine plasma concentrations predict virological failure in an unselected HIV-1-infected population. Clin. Pharmacokinet., 42: 599-605. PMID: 12793844

Wang, J., H. Kou, Q. Fu, Y. Han and Z. Qiu, 2011. Nevirapine plasma concentrations are associated with virologic response and hepatotoxicity in chinese patients with HIV infection. PLoS One, 6: 26739-26739. DOI: 10.1371/journal.pone.0026739

WHO, 2006. The World Health Report 2006: Working Together for Health. 1st Edn., World Health Organization, Geneva, ISBN-10: 241563176, pp: 209. 